\title{
VLF Waves Characterization: Wavelet Feature Extraction Method
}

\author{
Shivali Verma ${ }^{1}$, Harsha Jalori ${ }^{2}$, A. K. Gwal ${ }^{3}$ \\ ${ }^{1,3}$ Space Science Laboratory, Department of Physics Barkatullah University, Bhopal-462026, India \\ ${ }^{2}$ Government Science and Commerce College, Benazir, Bhopal-462026, India
}

\begin{abstract}
In an effort to provide a more efficient representation of the very low frequency (VLF) signal, the application of the wavelet analysis is considered. This research presents an effective and robust method for extracting features for VLF processing. Based on the time-frequency multi-resolution property of wavelet transform, the input VLF signal is decomposed into various frequency channels. The major issues concerning the design of this Wavelet based VLF recognition system are choosing optimal wavelets for VLF signals, decomposition level in the multilevel 1-D wavelet decomposition, selecting the feature vectors from the wavelet coefficients. More specifically classification of various VLF signals using the Wavelet decomposition is described. By this effort we find the different features for the various VLF signal
\end{abstract}

Keywords: Chorus, Feature extraction, Hiss, Very low frequency, Wavelet transforms, Whistler

\section{Introduction}

VLF emissions are natural radio phenomena, whose origin is from magnetospheric sources or in man-made sources such as VLF transmitters. For Space weather studies the investigation of VLF data provides an influential tool for remote sensing of the processes in the magnetosphere. In recent year the prediction of earthquake by VLF waves also the point of study. During the earthquake effect analysis two events are mainly under observation one is hiss and other is chorus (Rodge et al, 1999a), they are the important categories of magnetospheric VLF emission with whistler (Helliwell, 1965; Sazhin, 1982; Hayakawa et al., 1990; Sazhin and Hayakawa, 1992; Hattori and Hayakawa, 1994). Chorus are structured and discrete Its frequency and hiss are unstructured, on the other hand whistler are descending and ascending tones generated through the lighting discharge and which have propagated through the magnetosphere (Storey, 1953). They all have specific characteristics with respective time and frequency, one can be identified it by the spectrograms or by hearing its sound. Above mention studies need continues observation of this phenomena. It created the importance of managing a continuous flow of data through automation of the analysis and classification process. Automation is possible by the machine learning that required a specific representation and characterization. In the past many scientists were working on automatic detection of VLF emission (Buzzi,2006; Linchtenbeger et al., 2008; Ferencz et al., 2009; Golden et al., 2011;Ferencz et al., 2014) by analysis of the spectrogram of VLF signals. As we know that the VLF signals are non-stationary signals and spectrogram is constructed by using Short Time Fourier transform (STFT) in which signal segment within the window function is assumed to be stationary (Akay,1997). Therefore the spectrogram is not suitable for the characteristics of these VLF signals for the automatic signal classification system which is employed by Buzzi, 2006 for the whistler detection. In geophysical process the signals are non-stationary so for characterization of the signals scientist use wavelets transform (Kumar and Efi, 1994). The wavelet transform is a means that converts a signal into a different time frequency segment. This conversion reveals the characteristics hidden in the novel signal by noticing changes which arise rapidly in a signal as well as changes which occur over a longer duration in the signal. So for the characteristic and extraction of the feature we consider the possibility of providing a unified wavelet-based feature extraction tool which is designed to resist optimally with the whistler, hiss and chorus characteristics particular to VLF wave, in the most computationally efficient manner.

In an effort to offer a more effective representation of the very low frequency (VLF) emission, the privilege of the feature extraction based on an effective and robust method for extracting features for VLF signals. Feature extraction methodologies analyze signals to extract the most prominent features that are representative of the various classes of VLF signals. The main aim of feature extraction is to obtain the further information 's from the VLF signal based on the time-frequency multi-resolution property of wavelet transform; the input VLF signal is decomposed into various frequency channels. A major issue concerning the design of the VLF recognition system is choosing optimal wavelets for VLF signals, decomposition level in the multilevel 1-D wavelet decomposition, selecting the feature vectors from the wavelet coefficients. The Solution of this problem is also discus in the work. This method is very useful for the specifically automatic classification of various VLF signals using the Wavelet decomposition.

\section{Theoretical Consideration}

For the Feature extraction we employ audio parameter with discrete wavelet transform .Thus, the detail of both are given below:

\subsection{Discrete Wavelet Transform}

A function $\mathrm{f}(\mathrm{t})$ could be inscribed as a series expansion in terms of the scaling function and wavelets by (Steendam and Moeneclaey,2001) 
International Journal of Science and Research (IJSR)

ISSN (Online): 2319-7064

Index Copernicus Value (2013): 6.14 | Impact Factor (2014): 5.611

$\boldsymbol{f}(\boldsymbol{t})=$

$\sum_{p=p 0}^{\infty} \sum_{q=-\infty}^{\infty} a_{p 0}(q) \phi_{p, k}(t)+\sum_{p=p o}^{\infty} \sum_{q=-\infty}^{\infty} b_{p o}(q) \Psi_{p, q}(t)$

Where $\boldsymbol{\phi}_{\boldsymbol{p}, \boldsymbol{k}}(\boldsymbol{t})$ is known as scaling function and $\boldsymbol{\Psi}_{\boldsymbol{p}, \boldsymbol{q}}(\boldsymbol{t})$ denotes the wave function. In this development, the first summation offers a function that is a low resolution or coarse approximation of $f(t)$ at scale $p_{0}$.

Table1Feature vector after the applying SFS .S1, S2 ...S100 denotes the signal.SF11, SF12 ...SF17 represent the feature of S1 signal. Similarly SF21 to SF27 denotes the feature of signalS2and so on.F1 toF7 is depicted the feature number.

For every cumulative $p$ in the second summation, a higher or finer resolution function is summed, which improves details. The choice of $p_{0}$ sets selected those coarsest scale whose space is spanned by $\phi_{p 0, q}(t)$. The residual part function is spanned by the wavelets giving the high-resolution details of the function. The set of coefficients in the wavelet expansion signified by equation 1 is called the discrete wavelet transform(DWT) of the function $f(t)$. These wavelet coefficients, in certain conditions, can completely explain the original function, and in a way alike to Fourier series coefficients, can be used for analysis, approximation, filtering and description. If the scaling function is well performed, then at a high scale, samples of the signal are very near to the scaling coefficients. As state before, for well-mannered scaling or wavelet functions, the samples of a discrete signal can approximate the highest attainable scaling coefficients. It is revealed that the scaling and wavelet coefficients at scale $p$ are associated to the scaling coefficients at scale $(p+1)$ by the next two relations which given below

$$
\begin{aligned}
& \boldsymbol{a}_{p}(\boldsymbol{q})=\sum_{m} \boldsymbol{h}(\boldsymbol{n}-2 \boldsymbol{q}) \boldsymbol{a}_{p+1}(\boldsymbol{n}) \\
& \boldsymbol{b}_{p}(\boldsymbol{q})=\sum_{m} \boldsymbol{g}(\boldsymbol{n}-2 \boldsymbol{q}) \boldsymbol{b}_{p+1}(\boldsymbol{n})
\end{aligned}
$$

Here $\mathrm{h}$ and $\mathrm{g}$ indicates the low-pass and high-pass filters consistent to the coefficients $h(n)$ and $\mathrm{g}(\mathrm{n})$ respectively. By the DWT we decompose the VLF signals up to three level and get three details (D1, D2, D3) and one approximation (A3) coefficients

\subsection{Formation of feature vector}

After the DWT of VLF signals in order to improve the correctness in classification of signal, it is important to select good features that can capture all the characteristics of VLF signal. The VLF signals are audio-frequency phenomenon (Allcock,1957) therefore at this point one can be choose those feature which are quantify for audio signal characterization .Numerous feature are available which provide help for discrimination. But we used time- frequency domain features to reveal the maximum information for the formation of feature vector. We used those audio features which illustrated by Giannakopoulos and Pikrakis, 2014. We have use 13 parameter for the each decomposition level (DL). The detail description of audio feature is given in Table 2 for the one DL.
Table 1 Audio Parameter with mathematical formula

\begin{tabular}{|c|c|}
\hline Parameter Name & Mathematical Formula \\
\hline Zero Crossing Rate & $Z_{i}=\frac{1}{2} \sum_{\mathrm{n}=2}^{\mathrm{N}} \mid \operatorname{sgn}\left(\mathrm{x}(\mathrm{n})-\operatorname{sgn}(\mathrm{x}(\mathrm{n}-1)) \mid \frac{\mathrm{Fs}}{\mathrm{N}}\right.$ \\
\hline Short time energy & $E E=\sum_{p} P_{p}^{(l)} \cdot \log _{2} P_{p}^{(l)}$ \\
\hline Energy Entropy & $E=\frac{1}{N} \sum_{n=0}^{N-1} x_{n}^{2}$ \\
\hline Spectral centriod & $s c=\frac{\sum_{k=0}^{k-1} \mathrm{f}_{\mathrm{k}} \mathrm{X}_{\mathrm{k}}}{\sum_{k=0}^{K-1} X_{k}}$ \\
\hline Spectral Spread & $s s=\sqrt{\frac{\sum_{k=0}^{k-1} X_{k}\left(f_{k}-s c\right)^{2}}{\sum_{k=0}^{k-1} X_{k}}}$ \\
\hline Spectral Entropy & $S E=-\frac{\sum_{k=0}^{k-1} X_{k} \cdot \log \left(X_{k}\right)}{\log (K)}$ \\
\hline Spectral Flux & $\sum_{k=1}^{R_{k}} X_{k}^{2}=0.90 \sum_{k=1}^{K} X_{k}^{2}$ \\
\hline Spectral Roll off & $s f l u x=\sqrt{\sum_{k=0}^{K-1}\left(X_{k, t}-X_{k, t-1}\right)^{2}}$ \\
\hline MFCCs & $M F F C m=\sum_{j=0}^{J-1}\left\{\log \left(E_{J}\right) \cos \left[m_{m}^{\frac{\pi}{j}}\left(j-\frac{1}{2}\right)\right]\right\}$ \\
\hline
\end{tabular}

Using above parameter for the each decomposition we form a feature vector. The feature vector of the first D1 is shown in Table 3.In this way we have calculated the feature vector for the D2,D3and A3.Finally arrange them in the below given form

$$
F=\left[\begin{array}{l}
D F 1 \\
D F 2 \\
D F 3 \\
A F 3
\end{array}\right]
$$

Where the DF1 is a feature vector of D1, similarly DF2 and DF3 denotes the feature vector of D2 and D3 respectively and the feature vector of A3 is represent by the AF3.Thus dimension of $\mathrm{F}$ is 84 by 1 .

Table 2 Feature Vector for the first detail

\begin{tabular}{|c|c|c|c|}
\hline DLs & Feature No. & Feature Name & $\begin{array}{c}\text { Feature } \\
\text { Vector(DF1) }\end{array}$ \\
\hline & F1 & Zero Crossing Rate & \\
& F2 & Short time energy & \\
\cline { 2 - 3 } & F3 & Energy Entropy & \\
\cline { 2 - 3 } & F4 & Spectral centriod & \multirow{2}{*}{21 by 1} \\
\cline { 2 - 3 } & F5 & Spectral Spread & \\
\cline { 2 - 3 } & F6 & Spectral Entropy & \\
\cline { 2 - 3 } & F7 & Spectral Flux & \\
\cline { 2 - 3 } & F8 & Spectral Roll off & \\
\cline { 2 - 3 } & F9-F21 & MFCCs & \\
\hline
\end{tabular}

\subsection{Feature selection of VLF signals}

By the above discussion we get the 84 feature for characterization of VLF signals which contains many features that are either redundant or irrelevant and it is necessary to mute of them. Feature selection method reduced the dimension of the data due to that accuracy, predication performance and better understanding of the feature extraction method have improved (Guyon and Elisseeff,2003).These methods are not similar as the dimensionality reduction method. Because such methods creating new combinations of features, where as feature selection methods choose those features which are already present in data with high discrimination ability.

\section{Volume 5 Issue 2, February 2016}




\section{International Journal of Science and Research (IJSR) \\ ISSN (Online): 2319-7064 \\ Index Copernicus Value (2013): 6.14 | Impact Factor (2014): 5.611}

In this study Forward Sequential Feature Selection (SFS)(Doak, 1992) technique in a wrapper fashion is used for fine feature selection. Quadratic Discriminant Analysis (QDA) as a classifier model provides the support for MCE (misclassification error).These is done by holdout method and cross validation method.

The VLF wave dataset used in the proposed work have been explored from the DEMETER. In total we used 300 different signals, of $1.5 \mathrm{sec}$ with sampling frequency $40000 \mathrm{~Hz}$, split in three main categories chorus, hiss and whistler denoted as

Set A-chorus having 100 samples

Set B-Hiss having 100 samples

Set C-Whistler having 100 samples

We decompose each signal up to three levels by using Haar wavelet. We obtained VLF high dimensional data which have 84 rows and 100 columns. The data variable consists of 100 observations with 84 features. We apply forward sequential feature selection on 84 features. For that we divide data into a training set of size 270 and a test set of size of size 30. The training set is used to choose the features and to fit the QDA model, and the test set is hardened to evaluate the performance of the finally selected feature. During the feature selection procedure, to evaluate and to compare the performance of the each candidate feature subset, we apply stratified 10 -fold cross-validation to the training set. It stops when the minimum of the cross-validation MCE is found. The optimum feature set obtained after the completion of algorithm is depicted in Table 3 with general description.

\section{Results and Discussions}

In these work we have collected the chorus which is obtained from the latitude $\sim 55^{\circ}$ with frequency range about $<10 \mathrm{kHz}$, the hiss is consider here is mid-latitude hiss $(>3 \mathrm{kHz})$.

Table 3: Detail description of selected feature by the SFS to reduce the vector space

\begin{tabular}{|c|c|c|c|c|}
\hline \multirow{2}{*}{$\begin{array}{c}\text { Feature no } \\
\text { (Before } \\
\text { selection) }\end{array}$} & \multirow{2}{*}{$\begin{array}{l}\text { Feature no } \\
\text { (After } \\
\text { selection) }\end{array}$} & \multicolumn{3}{|c|}{ Feature Detail } \\
\hline & & $\overline{D L s}$ & $\begin{array}{c}\text { Frequency } \\
\text { range of DLs }\end{array}$ & Feature Name \\
\hline F4 & F1 & D1 & $20 \mathrm{kHz}-10 \mathrm{kHz}$ & Spectral Centriod \\
\hline F7 & F2 & D1 & $20 \mathrm{kHz}-10 \mathrm{kHz}$ & Spectral Flux \\
\hline F31 & F3 & D2 & $10 \mathrm{kHz}-5 \mathrm{kHz}$ & Second MFCC \\
\hline F48 & F4 & D3 & $5 \mathrm{kHz}-2.5 \mathrm{kHz}$ & Energy entropy \\
\hline F49 & F5 & D3 & $5 \mathrm{kHz}-2.5 \mathrm{kHz}$ & Spectral Flux \\
\hline F73 & F6 & A3 & $2.5 \mathrm{kHz}-0 \mathrm{kHz}$ & Second MFCC \\
\hline F74 & F7 & A3 & $2.5 \mathrm{kHz}-0 \mathrm{kHz}$ & Third MFCC \\
\hline
\end{tabular}

and ELF hiss $(<2 \mathrm{kHz})$ between the latitude $55^{\circ}$ to $40^{\circ}$ and the whistler(whistler train) are collect from the mid latitude.

In VLF spectrogram (Onishi et al., 2010) the VLF emission are describe special structured and occurs in different frequency band within $\sim 20 \mathrm{kHz}$.(Storey, 1953; Taylor and Gurnett, 1968; Dunckel and Helliwell, 1969; Sonwalkar, 1995).
Table 4: Feature vector of selected feature for the 100 VLF signals

\begin{tabular}{|c|c|c|c|c|c|c|c|}
\hline Signals /feature & S1 & S2 & S3 & S4 &. &. & S100 \\
\hline F1 & SF11 & SF21 & SF31 & SF41 &. &. & SF1001 \\
\hline F2 & SF12 & SF22 & SF32 & SF42 & &. & SF1002 \\
\hline F3 & SF13 & SF23 & SF33 & SF43 &. &. & SF1003 \\
\hline F4 & SF14 & SF24 & SF34 & SF44 &. &. & SF1004 \\
\hline F5 & SF15 & SF25 & S35 & S45 &. &. & SF1005 \\
\hline F6 & SF16 & SF25 & SF36 & SF46 &. &. & SF1006 \\
\hline F7 & SF17 & SF27 & SF37 & SF47 &. &. & SF1007 \\
\hline
\end{tabular}

Therefore it is must to study each frequency range for the characterization of VLF signals which is possible by the DWT. Accuracy of DWT based feature selection results depends on the choices of the mother wavelet. Hence we decompose D1 and D2level by Haar wavelet (Alfred,1910) and D3 and A3 through bio3.5(Charles, 1992) as it has maximum distribution energy at each DL for the VLF signal. After DWT we calculated different parameter with respected to the time and frequency for each DLs and form a feature vector of 7 by 1 dimension. This feature vector is shown in Table 4.

According to feature vector mention in Table 4, we calculated the feature vector of hiss, chorus and whistler by means of the 100 signals for each VLF emission. The feature vector of each emission is the new feature vector, able to discriminate the VLF signals. From Figure1, it is obvious that from feature number one to five, the shape of curve is same but the magnitude of each feature is different for each VLF emission plotted in Figure 1 (a), (b) and (c). From Table 3 we found that at least one feature is selected from each band which plays an important role for characterizing the VLF emissions. Because these bands shows variation in energy and shape with respect to time and frequency for the VLF signals.
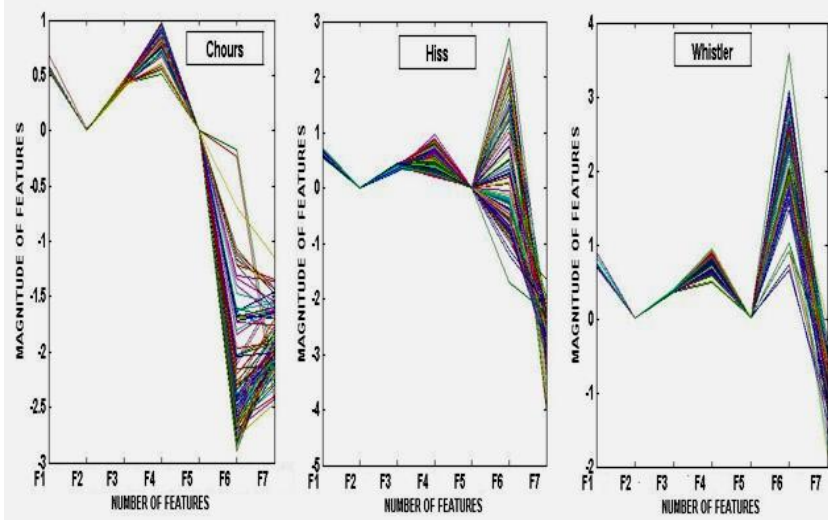

Figure 1: Feature vector plot of Chorus, Hiss and Whistler

during the occurrences of different VLF emission. On analyzing Figure 1 and Table 3 simultaneously, we get the following outcomes:

i)The first feature is selected from the frequency band 10 $\mathrm{kHz}-20 \mathrm{kHz}$ is Spectral Centriod (SC). This reveals the brightness of sound signal which helps to distinguish between sounds. Whistler have high value of SC as compared to the hiss and the chorus because most of the whistles are having their nose frequency and upper cutoff 


\section{International Journal of Science and Research (IJSR) \\ ISSN (Online): 2319-7064}

Index Copernicus Value (2013): 6.14 | Impact Factor (2014): 5.611

frequency in this range (Helliwel1, 1965) and sometimes the amplitude of whistler also approaches to maximum $\sim 15 \mathrm{kHz}$ (Helliwel1, 1965).On the other hand the hiss band have upper cutoff in this range but it is actually thermal noise (Gurnett, 1966) causing a low SC. The chorus is not reported in this frequency range (e.g. Sazhin and Hayakawa, 1992; Manninen et al., 2012).

ii)Spectral Flux (SF) is the second feature and its value for each VLF emission is very low. This illustrates that the local spectral change is very low with respect to the frequency, in frequency band $10 \mathrm{kHz}-20 \mathrm{kHz}$.In this frequency band only the upper cutoff frequency whistler(Carpenter, 1968) and hiss is present which are not showing much variation with respect the frequency.

iii)The third feature, second MFCC which is selected from the $10 \mathrm{kHz}-5 \mathrm{kHz}$ band. It shows value of the amplitude for lower frequency of this band $(\sim 6 \mathrm{kHz})$. For the whistler and the chorus value of MFCC is same for all signals but for the hiss variations are prevalent since its intensity varies with time and frequency (e.g., Gurnett, 1966; Sonwalkar and Inan, 1988). This band contains the lower cut off frequency of VLF hiss and its intensity is found to increase on enhancing frequency. Thus the MFCC values changes for the hiss only.

In frequency band $5 \mathrm{kHz}-2.5 \mathrm{kHz}$ we found that the value of Energy Entropy (EE) for all emission varies from signal to signal. EE is the signature of the distortion in the VLF signal relating to frequency. The whistler and the chorus are the structured emission (Storey, 1953;Sazhin and Hayakawa, 1992) and their frequency is either rise or fall in a particular manner with time. On the other hand hiss is incoherent emission which shows no obvious structure (e.g., Helliwell, 1965).Therefore chorus and whistler undergoes uniform distortion in signal instead of hiss.

iv) The frequency band, $5 \mathrm{kHz}-2.5 \mathrm{kHz}$ is an important band and the fifth feature which is selected in these range is SF. SF indicates the spectral change with frequency which is dominant in this range because all the components of VLF emissions are present here.

v) Last two features are selected from frequency band 2.5 $\mathrm{kHz}$ to few Hz. They are the second and third MFCC. The feature curve; shape is reverse for chorus as compare to hiss and whistler for this feature. Basically in this frequency range the lower cutoff frequency of whistler ELF hiss and the chorus components are generated (Helliwell, 1965). The selected features are related to the amplitude. When we analyse the curve which is formed by these two features, we found that the chorus show inverted curve as compare to the hiss and whistler, which indicates that the amplitude variations are same for the hiss and whistler. Hence we infer of the fact that the ELF hiss is generated due the lightning as whistler, whereas chorus is formed by high energy electrons (by acceleration) outside the plasma sphere (Horne et al., 2005).

These features are able to characterize the VLF emission is indentified by their classification ability Thus we trace a Andrews plot (details, Andrews, 1972) which offers the visual classification of this signals is revealed is Figure 2

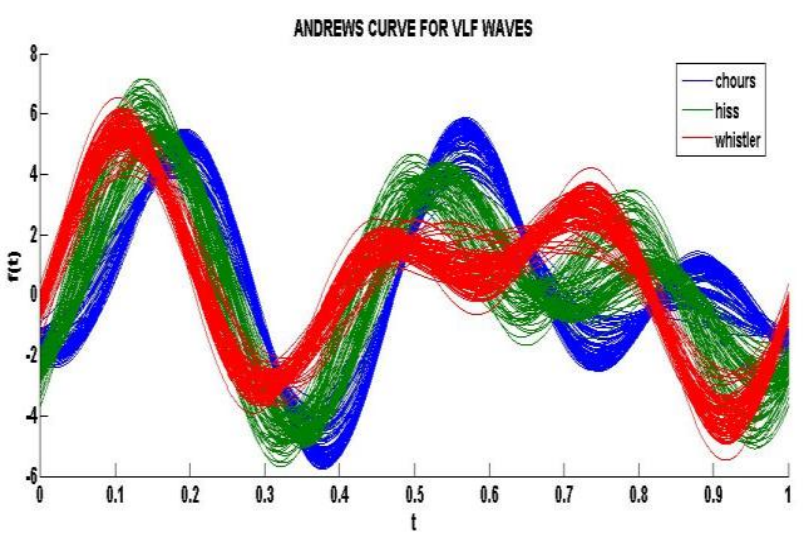

Figure 2: Andrews curve for the classification of VLF waves. Trace on the basis of selected seven feature. Red curve is denotes to the whistler, green denotes hiss and blue denotes the chorus.

The Andrews curve shows that these features are able to well classify the VLF wave because we can able to view each VLF emission clearly.

\section{Conclusions}

The characterization of the three main VLF emissions, the chorus, the hiss and the whistler is a crucial phase in machine learning .So in this chapter wavelet transform based techniques for feature extraction were performed. A wellselected feature set can result in quality representation of VLF wave whereas a wrongly chosen feature set can result in poor representation in machine learning. As VLF signals are non-stationary, the conventional method of frequency analysis is not highly successful in diagnostic presentation. Thus, it is important to reveal the all features which can discriminate the VLF signals in time and frequency domain. So an algorithm for characterization of VLF signal base on DWT and parameter of audio signals has been proposed for feature extraction. In absence of prior knowledge regarding the relevance of the individual features for successful recognition, as much information as possible is included into the feature vector. In addition this method not only provide better representation for the recognition of the three VLF signals-chorus, hiss and whistler also, it can reduce memory space, shorten pre-processing needs and increase computation speed for the machine learning of VLF signal. In future work we involve wave packet decomposition instead of the DWT for the feature extraction.

\section{References}

[1] Rodger, C. J., R. L. Dowden, and N. R. Thomson, Observations of electromagnetic activity associated with earthquakes by low-altitude satellites, in Atmospheric and Ionospheric Electromagnetic Phenomena Associated With Earthquakes, pp. 697-710, Terra Sci., Tokyo, 1999a

[2] Helliwell, R.A.: 1965, „Whistler and related ionospheric phenomenae, Stanford University Press, Stanford, California, USA.

[3] Sazhin, S.S. and Hayakawa, M.: 1992, „Magnetospheric chorus emissions: A reviewe, Planet. Space Sci., 40, 681697.

[4] Sazhin, S.S., Hayakawa, M. and Bullough, K.: 1992, „Whistler diagnostics of magnetospheric parameters, A 


\section{International Journal of Science and Research (IJSR) \\ ISSN (Online): 2319-7064}

Index Copernicus Value (2013): 6.14 | Impact Factor (2014): 5.611

review", Ann. Geophys., 10, 293-308.

[5] Hayakawa, M. and Sazhin, S.S.: 1992, „Mid-latitude and plasmaspheric hiss: A reviewe, Planet. Space Sci., 40, $1325-1338$.

[6] Hattori, K. and M. Hayakawa, Consideration of dynamic spectra and direction finding results of hiss-triggered chorus emissions, Proc. NIPR Symp. Upper Atmos. Phys., 7, 40-52, 1994.

[7] Storey, L.R.O.: 1953, „An investigation of whistling

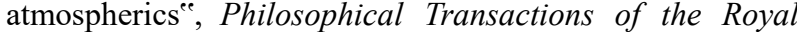
Society (London) A (246), 113-141.

[8] Platino, M., U. S. Inan, T. F. Bell, J. S. Pickett, E. J. Kennedy, J. G. Trotignon, J. L. Rauch, and P. Canu ,Cluster observations of ELF/VLF signals generated by modulated heating of the lower ionosphere with the HAARP HF transmitter, Ann. Geophys., 22(7), 2643-2653. 2004

[9] Lichtenberger, J., Cs. Ferencz, L. Bodnár, D. Hamar, and P. Steinbach, Automatic Whistler Detector and Analyzer system: Automatic Whistler Detector, J. Geophys. Res., 113, A12201, , 2008

[10] Ferencz, O.E., L. Bodnár, Cs. Ferencz, D. Hamar, J. Lichtenberger, P. Steinbach, V. Korepanov, G. Mikhaylova, Yu. Mikhaylov, and V. Kuznetsov, Ducted whistlers propagating in higher order guided mode and recorded on board of Compass- 2 satellite by the advanced Signal Analyzer and Sampler SAS2, J. Geophys. Res., 114, 2009.

[11] Golden, D.I., M. Spasojevic, and U.S. Inan, Determination of solar cycle variations of midlatitude ELF/VLF chorus and hiss via automated signal detection, J. Geophys. Res., $116,, 2011$.

[12] Csaba Ferencz,Georgii Lizunov, François Crespon, Ivan Price, Ludmil Bankov, Dorota Przepiórka, Klaus Brieß, Denis Dudkin, Andrey Girenko, Valery Korepanov, Andrii Kuzmych, Tetiana Skorokhod, Pencho Marinov, Olena Piankova, Hanna Rothkaehl, Tetyana Shtus, Péter Steinbach, János Lichtenberger, Arnold Sterenharz and Any Vassileva, Ionosphere Waves Service (IWS) - a problem-oriented tool in ionosphere and Space Weather research produced by POPDAT project Space Weather space clim.,A17,24,2014

[13] Buzzi A. DEMETER Satellite Data Analysis of SeismoElectromagnetic Signals, Doctorate Thesis, DEPARTMENT of PHYSICS, ROMA TRE University LPCE/CNRS Orl'eans University Co-Tutorship Agreement .Vinci. Programme of the Italian-French University,2006

[14] Akay M Wavelet applications in medicine IEEE Spectrum 34(5)50-56(ch6)

[15] N. Wyse, R. Dubes, and A. K. Jain. A critical evaluation of intrinsic dimensionality algorithms. In E. S. Gelsema and L. N. Kanal, editors, Pattern Recognition in Practice, pages 415-425. Morgan Kaufmann Publishers, Inc, San Mateo, CA, 1980 .

[16] Rafael C. Gonzalez, Richard E. Woods, Digital Image Processing (Second Edition), Prentice Hall, Upper Saddle River, NJ, 2002

[17] Y. Zheng and E. A. Essock, "Novel feature extraction method-Wavelet-Fourier analysis and its application to glaucoma classification," in Proceedings of 7th Joint Conference on Information Sciences, 2003, pp. 672-675.

[18] http://engineering.rowan.edu/ polikar/WAVELETS/WTtuto rial.html ; the Wavelet Tutorial by Robi Polikar.

[19] Debasis choudhury ,characterization of power quality disturbances using signal processing and soft computing techniques, thesis Department of Electrical Engineering National Institute of Technology Rourkela, 2013
[20] H. Steendam and M. Moeneclaey "The Effect of Carrier Frequency Offsets on Downlink and Uplink MC-DSCDMA", IEEE JOURNAL on Select. Areas in Comm., vol. 19 , no. 12 , Dec. 2001

[21] Ernst D. Schmitter Analysing and Classifying VLF Transients World Academy of Science, Engineering and Technology International Journal of Environmental, Chemical, Ecological, Geological and Geophysical Engineering Vol:1, No:6, 2007

[22] Kumar Praveen, Efi Foufoula-Georgiou, WAVELET ANALYSIS FOR GEOPHYSICAL APPLICATIONS, Reviews of Geophysics, 35, 4, 385-412, 1997

[23] Shukla, K. K. , Tiwari Arvind K.,Efficient Algorithms for Discrete Wavelet Transform With Applications to Denoising and Fuzzy Inference Systems springer Briefs in Computer Science ,2013

[24] Daniel T.L. Lee and Akio Yamamoto Wavelet analysis and theoretical application Hewlett-Packard Journal,1994.

[25] Ngui w.k.,Leong Salman M.,Lim Meng Hee Abdelrhman A.M.,Wavelet analysi :Mother selection methods Applied Mechanics and Materials Vol.393 ,953-958,2013.

[26] Allcock, G. M. (1957), A study of the audio-frequency radio phenomenon known as 'dawn chorus', Aust. J. Phys., 10, 286-298.

[27] Giannakopoulos Theodoros, Pikrakis Aggelos, Introduction to Audio Analysis: A MATLAB ${ }^{\circledR}$ Approach,book, Academic Press,2014

[28] Morsi W. G. and El-Hawary M. E., The most suitable Wavelet for steady-state power systemdistorted waveforms, Canadian Conference on Electrical and Computer Engineering. 17-22,2008.

[29] Golden Daniel I "source variation and ground accessibility of magnetosphericmid-latitude elf/vlfchorus and hiss",dissertation,Stanford University, 2011

[30] Chen, C. H., Signal processing handbook, Dekker, New York, 1988

[31] Scheier, E., Slaney, M., "Construction and evaluation of a robust multifeature speech/music discriminator," in Proc. IEEE Int. Conf. On Acoustics, Speech, and Signal Processing, ICASSP'97, pp. 1331-1334, 1997

[32] Grey J. M. and J. W. Gordon. Perceptual effects of spectral modi- fications on musical timbres. The Journal of the Acoustical Society of America, 63(5):1493-1500, 1978.

[33] Tzanetakis G. and P. Cook. Musical genre classification of audio signals. Acoustics Speech and Signal Processing (ICASSP), 2002 IEEE International Conference on, 10(5):293 - 302, 2002.

[34] Wang F. and H. Farid. Detecting re-projected video. In Kaushal Solanki, Kenneth Sullivan, and Upamanyu Madhow, editors, Information Hiding, volume 5284 of Lecture Notes in Computer Science, pages 72- 86. Springer Berlin Heidelberg, 2008.

[35] Guyon isabelle and Elisseeff Andre ,An Introduction to Variable and Feature Selection Journal of Machine Learning Research 3 (2003) 1157-1182

[36] Kohavi, R.; John, G.; Long, R.; Manley, D.; and Pfleger, K 1994. MLC++: A machine learning library in C++. In Tools with Artificial Intelligence, 740-743. IEEE Computer Society Press.

[37] Kohavi R.and John G H, Wrappers for feature subset selection artificial intelligence97(1-2),273-324,1997

[38] Dash, M., \& Liu, H. (1997). Feature selection for classifications. Intelligent Data Analysis: An International Journal, 1, 131-156.

[39] Doak, J. ,An evaluation of feature selection methods and their application to computer security Davis, CA: 


\section{International Journal of Science and Research (IJSR) \\ ISSN (Online): 2319-7064}

Index Copernicus Value (2013): 6.14 | Impact Factor (2014): 5.611

University of California, Department of Computer Science Technical Report CSE-92-18. 1992.

[40] Srivastava S., Gupta M.R., and Frigyik B.A. , Bayesian Quadratic Discriminant Analysis. The Journal of Machine Learning Research, 8:1277-1305, 2007.

[41] Hastie T., R. Tibshirani, and J. Friedman. The Elements of Statistical Learning. Springer-Verlag, New York, 2001.

[42] Kohavi1995, A study of cross-validation and bootstrap for accuracy estimation and model selection, in C. S. Mellish, ed., 'Proceedings of the 14th International Joint Conference on Artificial Intelligence', Morgan Kaufmann Publishers, Inc., pp. 1137--1143.

[43] Onishi, T. and Berthelier, J.-J.: Synthetic characterization of VLF electric field spectra from the ICE experiment on DEMETER: Automatic recognition and characterization of natural and man made emissions, Notes du pole de plan etologie, n. 21, June 2010.

[44] Taylor,W.W.L. and Gurnett,D.A.Morphology of VLF emissions observed with the Injun3 satellite .Journal of Geophysical Research 73,1968.

[45] Dunckel, N., and R. A. Helliwell (1969), Whistler-mode emissions on the OGO 1 satellite, J. Geophys. Res., 74(26), 6371-6385.

[46] Haar, Alfréd "Zur Theorie der orthogonalen Funktionensysteme", Mathematische Annalen 69 (3): 331371 (1910),

[47] Charles K. Chui, 1992. An Introduction to Wavelets, Academic Press, 1992.

[48] Burtis, W. J., and R. A. Helliwell (1976), Magnetospheric chorus: Occurrence patterns and normalized frequency, J. Geophys. Res., 24, 1007-1024.

[49] Burton, R. K., and R. E. Holzer (1974), The origin and propagation of chorus in the outer magnetosphere, J. Geophys. Res., 79, 1014-1023.

[50] Fraser-Smith, A. C., P. R. McGill, A. Bernardi, R. A. Helliwell, and M. E. Ladd (1991), Global measurements of lowfrequency radio noise, in Environmental and Space Electromagnetics, pp. 191 - 200, Springer, New York.

[51] Helliwell, R.A.: 1965, „Whistler and related ionospheric phenomena ${ }^{e e}$, Stanford University Press, Stanford, California, USA.

[52] Gurnett A.D. A satellite study of VLF hiss .Journal of geophysical research, Vol 71,5599-5615,1966.

[53] J. Manninen , N. G. Kleimenova , O. V. Kozyreva , M. Parrot, T. Raita, and T. Turunen Experimental evidence of the simultaneous occurrence of VLF chorus on the ground in the global azimuthal scale - from pre-midnight to the late morning Ann. Geophys., 30, 725-732, 2012

[54] Sazhin, S.S., Hayakawa, M. and Bullough, K.: 1992, „Whistler diagnostics of magnetospheric parameters, A reviewe, Ann. Geophys., 10, 293-308.

[55] Carpenter D.K. Ducted whistler -mode propagation in the magnetosphere a half-gyrofrequency upper intensity cutoff and some associated wave growth phenomena Journal of Geophysical Research ,Space Physics ,Vol.73,291929281968

[56] Sonwalkar S. V. and Inan S. U. Wave normal direction and spectral properties of whistler mode hiss observed on the DE1 satellite Journal of geophysical research, Vo193, 74937514,1988

[57] Horne, R.B., Thorne, R.M., Shprits, Y.Y., Meredith, N.P., Glauert, S.A., Smith, A.J., Kanekal, S.G., Baker, D.N., Engebretson, M.J., Posch, J.L., Spasojevic, M., Inan, U.S., Pickett, J.S. and Decreau, P.M.E.: 2005, „Wave acceleration of electrons in the Van Allen radiation belts "e, Nature, 437, 227-230, doi:10.1038/nature03939.
[58] Andrews, D. F. (1972). Plots of high dimensional data. Biometrics, 28, 125-136

[59] Graps,A "An Introduction to Wavelets", IEEE Computational Science and Eng., Vol. 2, No. 2, 1995.

[60] Goswami J. C., Chan A. K., "Fundamentals of Wavelets Theory, Algorithms and Applications", John Wiley \& Sons Ltd. 1999.

[61] Mallat S., "A Theory for Multiresolution Signal Decomposition: the Wavelet Representation, " IEEE Pattern Anal. And Machine Intel, vol. 11, no. 7, pp. 674693. 1989.

\section{Author Profile}

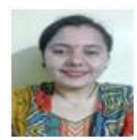

Shivali Verma did her M.Sc. (Physics) from the Govt. Motilal Vigyan Mahavidyalaya, Bhopal. She is having 16year teaching experience. Her research area is Space and Plasma Physics.

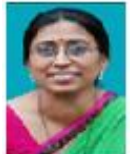

Dr. Harsha Jalori working as Astt. Prof in Govt Science and Commerce College, Benazir, Bhopal. She did her Ph.D. from the Barkatullah University,Bhopal in 2000. She is having the 30 year teaching experience. Her research area is Space and Plasma Physics. She in the member of Plasma Society of India and Indian national science Congress. She published large number of research paper. Till date she has successfully guided 3 in the related areas of research.

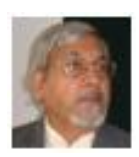

Dr. A. K. Gwal has done M.Sc. (Physics) in 1970 from Banaras Hindu University, Varanasi. Consequently, he was awarded Ph.D. degree in Physics in 1977 for his scientific contribution in the area of Space and Plasma Physics. In continuation, he worked as a post doctoral fellow in the same field. In 1979, he joined Indian Institute of Technology, Kharagpur as a Scientific Officer and worked in the area of Antenna theory and designs. In 1981, he left for Canada on a Visiting Scientist Fellowship of Natural Science and Engine Canada, where he was involved in the problems of plasma instabilities and their applications in the magnetosphere using satellite data. On return from abroad in 1983, he joined Indian Institute of Technology, Delhi, as a Pool Scientist (C. materials till 1987. In 1987, he joined Barkatullah University, Bhopal as a lecturer. He established the Space Science Laboratory in this department. Subsequently, he became Reader in the year 1991 and Professor in 1999. . Dr. Gwal is a member of various scientific societies, viz. Indian Physics Association, Plasma Science Society of India and American Geophysical Union (USA). To his credit he has several distinctions, abroad visits. He has published 72 papers in various national/ international authored 03 books. His elaborationse and the large number of research projects being handled by him. Till date he has successfully guided 12 in the related areas of research. 